\title{
OXA-235, a Novel Class D $\beta$-Lactamase Involved in Resistance to Carbapenems in Acinetobacter baumannii
}

\author{
Paul G. Higgins, ${ }^{a}$ Francisco J. Pérez-Llarena, ${ }^{\mathrm{b}}$ Esther Zander, ${ }^{\mathrm{a}}$ Ana Fernández, ${ }^{\mathrm{b}}$ Germán Bou, ${ }^{\mathrm{b}}$ Harald Seifert ${ }^{\mathrm{a}}$ \\ Institute for Medical Microbiology, Immunology and Hygiene, University of Cologne, Cologne, Germanya; Servizo de Microbioloxía-INIBIC, Complexo Hospitalario \\ Universitario A Coruña, Coruña, Spain ${ }^{\text {b }}$
}

We investigated the mechanism of carbapenem resistance in 10 Acinetobacter baumannii strains isolated from the United States and Mexico between 2005 and 2009. The detection of known metallo- $\beta$-lactamase or carbapenem-hydrolyzing oxacillinase (OXA) genes by PCR was negative. The presence of plasmid-encoded carbapenem resistance genes was investigated by transformation of A. baumannii ATCC 17978. Shotgun cloning experiments and sequencing were performed, followed by the expression of a novel $\beta$-lactamase in A. baumannii. Three novel OXA enzymes were identified, OXA-235 in 8 isolates and the amino acid variants OXA-236 (Glu173-Val) and OXA-237 (Asp208-Gly) in 1 isolate each. The deduced amino acid sequences shared 85\% identity with OXA-134, 54\% to 57\% identities with the acquired OXA-23, OXA-24, OXA-58, and OXA-143, and 56\% identity with the intrinsic OXA-51 and, thus, represent a novel subclass of OXA. The expression of OXA-235 in A. baumannii led to reduced carbapenem susceptibility, while cephalosporin MICs were unaffected. Genetic analysis revealed that $b l a_{\mathrm{OXA}-235}, b l a_{\mathrm{OXA}-236}$, and $b l a_{\text {OXA-237 }}$ were bracketed between two ISAbal insertion sequences. In addition, the presence of these acquired $\beta$-lactamase genes might result from a transposition-mediated mechanism. This highlights the propensity of $A$. baumannii to acquire multiple carbapenem resistance determinants.

cinetobacter baumannii is a nosocomial pathogen that is characterized by its innate and acquired antimicrobial resistance (1-3). Of major concern is the increased incidence of carbapenem resistance, which has risen dramatically over the last decade (1, 4, 5). A. baumannii carbapenem resistance is most frequently mediated through the presence of a carbapenemase (6). Metalloenzymes, such as VIM, IMP, and NDM, are still rare in this species, with the majority of reported carbapenem resistance mediated through the action of carbapenem-hydrolyzing class D $\beta$-lactamases (CHDLs) (2, 7-9). The CHDLs, also called oxacillinases (OXA), exhibit weak hydrolysis of carbapenems. However, the genes encoding OXA are often associated with insertion sequences that provide strong promoters, leading to overexpression and carbapenem resistance (7).

There are currently five subclasses of OXA associated with $A$. baumannii; the intrinsic chromosomal OXA-51-like, of which there are over 70 variants, and the acquired OXA-23-like, OXA-24 (OXA-40-like), OXA-58-like, and OXA-143-like (9). Acquired OXA are found both chromosomally and on plasmids and can be detected by multiplex PCR $(10,11)$. Here, we describe a novel OXA subclass identified in A. baumannii strains that could not be detected by previous PCR methods.

\section{MATERIALS AND METHODS}

Bacterial strains and antimicrobial susceptibility. Nine A. baumannii clinical isolates from the United States and one isolate from Mexico were included. Dates and places of isolation are shown in Table 1. Species identification was confirmed by gyrB multiplex PCR (12). All isolates were tested for their susceptibility to imipenem and meropenem by Etest (bioMérieux, Nürtingen, Germany), following the manufacturer's instructions. Isolates were typed by repetitive-sequence-based PCR (DiversiLab rep-PCR) as previously described and assigned to international clones using our in-house database $(8,13)$.

PCR experiments. Detection of the genes encoding known acquired CHDLs $\left(b l a_{\text {OXA-23 }}, b l a_{\text {OXA-24 }}, b l a_{\text {OXA-51 }}, b l a_{\text {OXA-58 }}\right.$, and $\left.b l a_{\text {OXA-143 }}\right)$ and metallo- $\beta$-lactamases ( $b l a_{\mathrm{IMP}}, \quad b l a_{\mathrm{VIM}}, \quad b l a_{\mathrm{GIM}}, b l a_{\mathrm{SPM}}, b l a_{\mathrm{SIM}}$, and bla $\left._{\mathrm{NDM}}\right)$ was performed as previously described $(10,11,14,15)$. The $b l a_{\text {OXA-51-like }}$ gene was amplified and sequenced using primers OXA69A and OXA69B, and the presence of the insertion sequence ISAbal upstream from it was investigated by $\operatorname{PCR}(7,16)$.

Plasmid analysis and cloning. To determine if carbapenem resistance was plasmid encoded, plasmid DNA was extracted from all the A. baumannii isolates listed in Table 1 using a commercial kit (Qiagen, Hilden, Germany) and used to transform electrocompetent A. baumannii ATCC 17978 as previously described for Pseudomonas aeruginosa (17). Selection was performed with ticarcillin at $100 \mu \mathrm{g} / \mathrm{ml}$. Reduced carbapenem susceptibility of transformants was tested by disc diffusion.

Plasmids that successfully conferred reduced carbapenem susceptibility when transformed in ATCC 17978 were used for shotgun cloning. Briefly, plasmid DNA was extracted, digested with the restriction enzyme EcoRI, ligated into EcoRI-cut pBBR1MCS (18), and used to transform Escherichia coli NEB5 $\alpha$ (New England BioLabs, Germany). Transformants were selected on LB agar plates containing $25 \mu \mathrm{g} / \mathrm{ml}$ ticarcillin, and the recombinant plasmid pOXA-235 was retained for further analysis. The size of plasmid inserts was determined by restriction analysis. Replicon typing of plasmids was performed as previously described (19).

To confirm the plasmid/chromosomal location of bla $a_{\text {OXA-235-like, }}$ DNA-DNA hybridization was performed using total DNA preparations (Qiagen) and the hybridized DNA separated by electrophoresis and transferred onto Hybond $\mathrm{N}+$ membranes. The location of $b l a_{\text {OXA-235-like }}$ was assessed by hybridization of digoxigenin (DIG)-labeled probes (Roche,

Received 6 December 2012 Returned for modification 1 January 2013 Accepted 15 February 2013

Published ahead of print 25 February 2013

Address correspondence to Paul G. Higgins, paul.higgins@uni-koeln.de. Supplemental material for this article may be found at http://dx.doi.org/10.1128 /AAC.02413-12

Copyright $\odot$ 2013, American Society for Microbiology. All Rights Reserved. doi:10.1128/AAC.02413-12 
TABLE 1 Acquired OXA and strain information

\begin{tabular}{|c|c|c|c|c|c|c|c|}
\hline Strain & $\begin{array}{l}\text { Date of isolation } \\
\text { (mo-yr) }\end{array}$ & $\begin{array}{l}\text { Type of } \\
\text { specimen }\end{array}$ & $\begin{array}{l}\text { Location where } \\
\text { isolate was recovered }\end{array}$ & $\begin{array}{l}\text { Acquired } \\
\text { OXA }\end{array}$ & $\begin{array}{l}\text { Bacterial location of acquired } \\
b l a_{\text {OXA }} \text { gene }\end{array}$ & $\begin{array}{l}\text { OXA-51-like } \\
\text { variant }\end{array}$ & $\begin{array}{l}\text { International } \\
\text { clone }^{a}\end{array}$ \\
\hline BMBF 255 & 06-2005 & Wound & $\mathrm{USA}^{c}$ & 236 & Chromosome and plasmid & 241 & Unc \\
\hline AF 684 & 03-2007 & $\mathrm{TS}^{b}$ & California & 237 & Plasmid & 66 & 2 \\
\hline AF 670 & 06-2007 & Blood & Arizona & 235 & Chromosome and plasmid & 242 & Unc \\
\hline AF 401 & 07-2007 & Small intestine & Mexico $^{d}$ & 235 & Chromosome and plasmid & 65 & 5 \\
\hline AF 667 & $10-2007$ & Wound & Arizona & 235 & Chromosome and plasmid & 242 & Unc \\
\hline AF 785 & $10-2007$ & TS & Nevada & 235 & Chromosome & 66 & 2 \\
\hline AF 678 & $12-2007$ & Sputum & California & 235 & Chromosome & 66 & 2 \\
\hline AF 707 & 01-2008 & Wound & Illinois & 235 & Chromosome & 66 & 2 \\
\hline AF 673 & 03-2008 & Sputum & Arizona & 235 & Chromosome & 66 & 2 \\
\hline AF 875 & $11-2009$ & Sputum & Utah & 235 & Chromosome & 66 & 2 \\
\hline
\end{tabular}

${ }^{a}$ As determined by rep-PCR and $b l a_{\mathrm{OXA}-51}$ typing. Unc, unclustered.

${ }^{b}$ TS, tracheal secretion.

${ }^{c}$ State not known.

${ }^{d}$ Isolate recovered from unknown location in Mexico.

Mannheim, Germany) specific for $b l a_{\text {OXA-235-like: Chromosomal location }}$ was shown by colocalization with a $b l a_{\text {OXA-51-like }}$ probe.

Novel $b l a_{\text {OXA-235 }}$ was cloned into the shuttle vector pWH1266 with its native promoter. Additionally, $b l a_{\text {OXA-235 }}$ and $b l a_{\text {OXA-24 }}$ were cloned into shuttle vector pETRA, expressed using the CTX-M14 promoter, and transformed into Acinetobacter baylyi and A. baumannii ATCC 17978 for antibiotic susceptibility testing (20).

DNA sequencing. Recombinant strain E. coli NEB5 $\alpha$ (pOXA-235) was extracted and sequenced by primer walking. A novel $b l a_{\text {OXA }}$ was identified by BLAST query. Primers specific for the new $b l a_{\mathrm{OXA}}$ (forward, $5^{\prime}$-CAAGCCATGCAAGCTTCT- ${ }^{\prime}$, and reverse, $5^{\prime}$-GCTGGACTTGAG GATCAAAG-3') were designed and used to screen the remaining 9 isolates. To confirm sequences of novel $b l a_{\mathrm{OXA}}$ variants, PCR and sequencing reactions were repeated using Phusion hot-start high-fidelity DNA polymerase (Thermo Fisher Scientific, Schwerte, Germany). Products were purified using the QIAquick PCR purification kit (Qiagen) and sequenced. Novel sequences were assigned numbers by the Lahey $\beta$-lactamase database (http://www.lahey.org/Studies/) and submitted to GenBank.

$\boldsymbol{\beta}$-Lactamase purification. To purify OXA-235, the $b l a_{\text {OXA-235 }}$ gene was cloned into the pGEX-6P-1 vector with the following primers: $5^{\prime}$-AA AAGAATTCTTGCCTGTTTCAAATTCG-3' (forward) and 5' -AAAAGT CGACTTACCCTTCAGCTTTCGG-3' (reverse), which generated the restriction sites EcoRI and SalI, respectively. Similarly, $b l a_{\text {OXA-24 }}$ that was previously cloned into the same vector was used as a comparator CHDL for kinetic studies (21). The constructs were transformed into E. coli BL21 and produced a fusion protein between glutathione $S$-transferase (GST) and the OXA-235/OXA-24 enzyme. The $\beta$-lactamase was purified to homogeneity according to the manufacturer's instructions for the GST gene fusion system (Amersham Pharmacia Biotech Europe $\mathrm{GmbH}$ ). After cleavage of GST from OXA-235/OXA-24, the purified protein ( $\geq 99 \%$ pure) appeared in SDS-PAGE as a single band (data not shown).

Determination of kinetic parameters. Ampicillin and oxacillin powders were purchased from Sigma Chemical Co. (St. Louis, MO). Imipenem and meropenem powders were gifts from Merck (Whitehouse Station, NJ) and AstraZeneca (London, United Kingdom), respectively. Nitrocefin was obtained from Unipath Oxoid (Basingstoke, Hants, United Kingdom), and isopropyl- $\beta$-D-thiogalactopyranoside (IPTG) was purchased from Roche (Basel, Switzerland). Hydrolysis of the antibiotics by the OXA-235 $\beta$-lactamase was monitored by recording the variation in absorbance resulting from the opening of the $\beta$-lactam ring under the following conditions: the antibiotic extinction coefficients for nitrocefin, ampicillin, oxacillin, imipenem, and meropenem were $+15,000,-820$, $+440,-9,000$, and $-6,500 \mathrm{M}^{-1} \mathrm{~cm}^{-1}$, respectively, and the wavelengths used were $260 \mathrm{~nm}$ for oxacillin, $482 \mathrm{~nm}$ for nitrocefin, $300 \mathrm{~nm}$ for imipenem and meropenem, and $235 \mathrm{~nm}$ for ampicillin. The tests were re- peated three times in phosphate-buffered saline (PBS) with $20 \mathrm{mg}$ BSA/ liter at $25^{\circ} \mathrm{C}$. The kinetic parameters for nitrocefin were determined from the initial rates by Hanes-Woolf linearization of the Henri-MichaelisMenten equation. For the other antibiotics, the $K_{m}$ value was measured as the $K_{i}$ in a competition experiment, with nitrocefin as the reporter substrate (22). The $k_{\text {cat }}$ values were then obtained by monitoring hydrolysis of the antibiotic at a concentration of $>10$ times the $K_{m}$.

Multiplex PCR to detect $\boldsymbol{b l a x A - 2 3 5 - l i k e}$. In order to detect $b l a_{\text {OXA-235-like }}$ by PCR, we designed primers to amplify $b l a_{\text {OXA-235 }}$, $b l a_{\text {OXA-236, }}$ and $b l a_{\text {OXA-237. }}$ Primer pair OXA-235_F ( $5^{\prime}$-TTGTTGCCTT TACTTAGTTGC- $\left.3^{\prime}\right)$ and OXA-235_R (5' -CAAAATTTTAAGACGGAT CG-3') were tested by addition to the existing OXA multiplex PCR and amplified using the published parameters $(10,11)$. The multiplex PCR was tested against the 10 bla $_{\text {OXA-235-like }}$ isolates listed in Table 1 , as well as previously characterized $A$. baumannii isolates possessing $b l a_{\mathrm{OXA}-51-\text { like }}$ and the acquired $b l a_{\text {OXA-23-like, }}, b l a_{\text {OXA-24-like, }} b l a_{\text {OXA-58-like, }}$ and $b l a_{\text {OXA-143-like, }}$ as previously described (10).

Nucleotide sequence accession numbers. The nucleotide sequences of the novel $b l a_{\text {OXA }}$ variants reported in this paper have been submitted to the EMBL/GenBank database under accession numbers JQ820240 (OXA235), JQ820242 (OXA-236), JQ820241 (OXA-237), JX025021 (OXA241), and JX025022 (OXA-242).

\section{RESULTS}

Antibiotic susceptibility, plasmid analysis, and PCR experiments. The 10 isolates were confirmed as carbapenem-resistant $A$. baumannii, recording imipenem and meropenem MICs of $\geq 16$ $\mu \mathrm{g} / \mathrm{ml}$. By PCR, they were negative for known CHDLs and metallocarbapenemases, and ISAbal was not adjacent to $b l a_{\text {OXA-51-like }}$. Sequencing of $b l a_{\text {OXA-51-like }}$ revealed that six isolates possessed OXA-66, which is characteristic of international clone 2 (IC2) (23). Typing by rep-PCR revealed that five of the IC2 isolates showed $\geq 99 \%$ similarity in their rep-PCR fingerprints, which suggests clonal spread of a strain into Utah, Illinois, California, and Nevada (see Fig. S1 in the supplemental material). Two novel OXA-51-like enzymes were identified in unclustered isolates, OXA-241 and OXA-242 (Table 1). Strains AF667 and AF670 were 98.9\% identical by rep-PCR and did not cluster with any isolate from our database.

Four isolates yielded plasmids that successfully transformed $A$. baumannii ATCC 17978 to reduced carbapenem susceptibility; these transformants exhibited phenotypic heterogeneous resistance that was characterized by a reduced inhibition zone compared to the inhibition zone of the untransformed control, within 


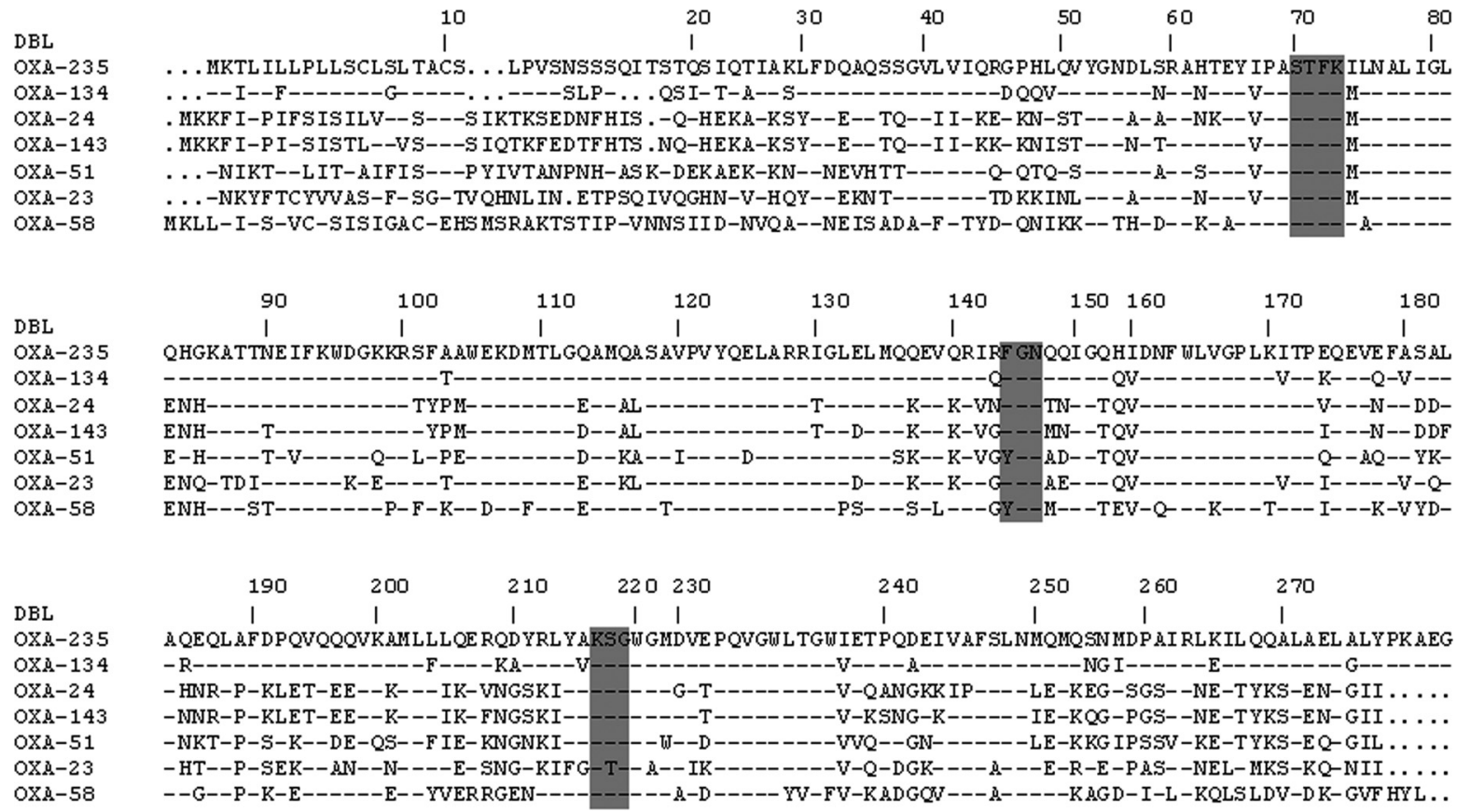

FIG 1 Alignment of the OXA-235 amino acid sequence with those of OXA-23, OXA-24, OXA-51, OXA-58, OXA-134, and OXA-143. Conserved residues are shaded. Dashes represent conserved amino acids. $\beta$-Lactamases are numbered according to the DBL numbering system (24).

which individual colonies were visible growing up to the imipenem disc. We did not detect cotransfer of other antibiotic resistance determinants, with the transformants showing no difference in their MICs to tetracycline, gentamicin, chloramphenicol, ciprofloxacin, or minocycline compared to the MICs for the untransformed parent strain (data not shown). The remaining 6 isolates that did not transform ATCC 17978 were presumed to have a chromosome-located resistance mechanism.

Identification of the OXA-235, -236, and -237 CHDLs. The transforming plasmids were used for shotgun cloning. EcoRI-restricted plasmid DNA from A. baumannii AF401 cloned into pBBR1MCS, followed by expression in E. coli, gave E. coli (pOXA235). Analysis of shotgun-cloned plasmid DNA revealed a $2.5-\mathrm{kb}$ insert which was sequenced by primer walking. DNA sequence analysis of plasmid pOXA-235 identified an 831-bp open reading frame (ORF) that encoded a novel class D $\beta$-lactamase of 276 amino acids (Fig. 1), which was assigned as OXA-235 by the $\beta$-lactamase database. Within the deduced protein sequence, a serinethreonine-phenylalanine-lysine tetrad (STFK) was found (24). A KSG element (positions 216 to 218) was present, as found in OXA24, OXA-51, OXA-58, and OXA-143 CHDLs, whereas a KTG motif is present in the OXA-23 enzyme and in most class D $\beta$-lactamases lacking any carbapenemase activity. The class D $\beta$-lactamase structural element FGN at positions 70 to 73 (DBL numbering) was conserved in OXA-235, as has been found in all of the CHDL sequences identified in A. baumannii, with the exception of

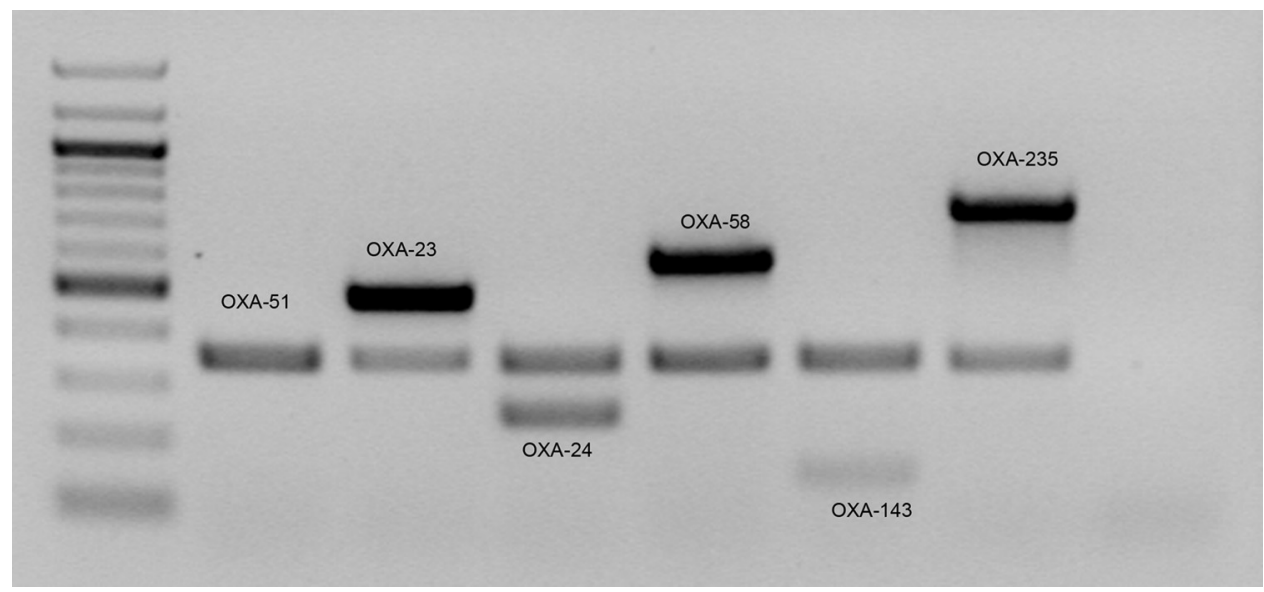

FIG 2 OXA-type multiplex PCR with additional OXA-235-like primers. The Acinetobacter baumannii isolates contain alleles encoding the intrinsic OXA-51-like (lanes 2 to 7 ) and the acquired OXA-23-like, OXA-24-like, OXA-58-like, OXA-143-like (lanes 3 to 6), and OXA-235 (lane 7). The molecular size marker (lane 1 ) is a 100-bp ladder (NEB, Frankfurt am Main, Germany). 
TABLE 2 MICs of A. baylyi and A. baumannii ATCC 17978 transformed with pETRA::OXA-24 and pETRA::OXA-235

\begin{tabular}{|c|c|c|c|c|c|c|}
\hline \multirow[b]{3}{*}{ Antibiotic } & \multicolumn{6}{|c|}{ MIC $(\mu \mathrm{g} / \mathrm{ml})$ of indicated strain transformed with: } \\
\hline & \multicolumn{2}{|l|}{ pETRA $^{a}$} & \multicolumn{2}{|l|}{ OXA-24 } & \multicolumn{2}{|l|}{ OXA-235 } \\
\hline & $>A$. baylyi & A. baumannii 17978 & A. baylyi & A. baumannii 17978 & A. baylyi & A. baumannii 17978 \\
\hline Imipenem & 0.125 & 0.25 & 32 & $>32$ & 1 & 3 \\
\hline Meropenem & 0.25 & 0.5 & 32 & $>32$ & 2 & 4 \\
\hline Ampicillin & 8 & 256 & $>256$ & $>256$ & $>256$ & $>256$ \\
\hline Cefoxitin & 24 & $>256$ & 24 & $>256$ & 16 & $>256$ \\
\hline Ceftazidime & 4 & 6 & 4 & 6 & 4 & 6 \\
\hline Cefotaxime & 6 & 16 & 8 & 16 & 8 & 16 \\
\hline Piperacillin & 6 & 24 & 256 & $>256$ & 48 & $>256$ \\
\hline $\mathrm{TZP}^{b}$ & 6 & 16 & 256 & $>256$ & 256 & 256 \\
\hline
\end{tabular}

${ }^{a}$ pETRA, plasmid alone as negative control.

${ }^{b} \mathrm{TZP}$, piperacillin-tazobactam.

those of the OXA-51 and OXA-58 subclasses (25). Also, OXA-235 showed key residues for carbapenem hydrolysis, $\mathrm{F}$ or $\mathrm{Y}$ at 112 and Met-223, previously identified in OXA-24, which define a tunnellike entrance for carbapenems to the active site (21).

The deduced amino acid sequence showed the greatest homology to OXA-134 (85\% identity), an intrinsic oxacillinase of $A$. lwoffii (26). The amino acid identities with the acquired CDHLs OXA-23, OXA-24, OXA-58, and OXA-143 were 54\% to 57\%, and the amino acid identity with OXA-51 was $56 \%$ (Fig. 1). Primer walking also revealed that $b l a_{\text {OXA-235 }}$ was bracketed by two copies of ISA ba1. Primers were designed to amplify $b l a_{\text {OXA-235 }}$, and these were used to screen the remaining 9 isolates. All isolates tested positive for the presence of $b l a_{\text {OXA-235 }}$ and ISAbal bracketing the gene. The PCR products were subsequently sequenced. Seven isolates were found to harbor OXA-235. Two novel variants of OXA235 were also identified, OXA-236 (Glu173-Val) and OXA-237 (Asp208-Gly) (Table 1). Multiplex PCR with primer pair OXA235_F/OXA-235_R amplified a 768-bp fragment that was easily distinguishable from the other $b l a_{\mathrm{OXA}}$ within the current OXA multiplex PCR (Fig. 2).

DNA-DNA hybridization confirmed a plasmid location in 4 strains; however, in three of these strains, a second positive signal was visible that corresponded to genomic DNA (colocalized with $\left.b l a_{\text {OXA-51-like }}\right)$. A 5 th strain (BMBF 255) showed a positive signal for chromosomal and plasmid location of $b l a_{\text {OXA-235-like }}$, but this plasmid failed to transform in ATCC 17978. PCR of extracted plasmid DNA confirmed these results. Two $b l a_{\text {OXA-235-positive }}$ plasmids were typeable using the method described by Bertini et al. (19), belonging to replicon groups 2 and 6 (originating from strains AF 401 and AF 678, respectively).

Transformation of bla $a_{\text {OXA-235 }}$ into A. baylyi and A. baumannii
ATCC 17978 led to reduced carbapenem susceptibility (Table 2). Cephalosporin MICs were unaffected. Similarly, transformation of A. baumannii ATCC 17978 with pWH1266-OXA-235 resulted in raising imipenem and meropenem MICs from 0.125 to $6 \mu \mathrm{g} / \mathrm{ml}$ and from 0.19 to $16 \mu \mathrm{g} / \mathrm{ml}$, respectively (data not shown). As seen with transformation with native plasmids, the transformants exhibited phenotypic heterogeneous resistance.

Kinetic determination of $k_{\text {cat }}, K_{m}$, and $k_{\text {cat }} / K_{m}$ against oxacillin, nitrocefin, ampicillin, meropenem, and imipenem was carried out (Table 3). The results of the kinetic analysis show that OXA-24 has an approximately 1,000 -times-lower $k_{\text {cat }} / K_{m}$ to oxacillin than to OXA-235, which indicates that OXA-235 has more oxacillinase activity than OXA-24. Conversely, there was a higher activity of OXA-24 with respect to carbapenems, with this enzyme having around 5 times more catalytic efficacy than OXA-235 for meropenem and imipenem. The kinetic results are consistent with the MIC data (Table 2). Despite the low catalytic activity of OXA-235, the enzyme exhibits sufficient hydrolysis to reduce carbapenem susceptibility when it is expressed in shuttle plasmid pETRA or pWH1266 in A. baylyi and A. baumanii.

\section{DISCUSSION}

Three novel carbapenem resistance determinants that were undetected using established PCRs have been identified in A. baumannii. These CHDLs, OXA-235, OXA-236, and OXA-237, were identified in isolates that originated primarily from the United States, but one isolate was recovered in Mexico. Thus, there are similarities to OXA-143-like, which has so far only been reported in Brazil $(27,28)$, and contrasts with OXA-23, OXA-24, and OXA-58, which, although first identified in Europe (Scotland, Spain, and France, respectively) $(25,29,30)$, have now been described glob-

TABLE 3 Kinetic data for purified OXA-235 and OXA-24 $\beta$-lactamases

\begin{tabular}{|c|c|c|c|c|c|c|}
\hline \multirow[b]{2}{*}{ Antibiotic } & \multicolumn{2}{|c|}{$k_{\text {cat }}\left(\mathrm{s}^{-1}\right)[$ mean $( \pm \mathrm{SD})]$} & \multicolumn{2}{|c|}{$\left.K_{m(\mu} \mathrm{M}\right)[$ mean $( \pm \mathrm{SD})]$} & \multicolumn{2}{|c|}{$k_{\mathrm{cat}} / K_{m}\left(\mu \mathrm{M}^{-1} \mathrm{~s}^{-1}\right)$} \\
\hline & OXA-24 & OXA-235 & OXA-24 & OXA-235 & OXA-24 & OXA-235 \\
\hline Nitrocefin & $\mathrm{NT}^{b}$ & $65.64( \pm 7)$ & NT & $53( \pm 7)$ & NT & 1.23 \\
\hline Ampicillin & $223( \pm 92.9)$ & $0.73( \pm 0.015)$ & $80( \pm 15)$ & $85( \pm 21)$ & 2.7 & 0.0085 \\
\hline Meropenem & $0.37( \pm 0.1)$ & $0.117( \pm 0.023)$ & $0.0099( \pm 0.0014)$ & $0.0159( \pm 0.0027)$ & 37.37 & 7.35 \\
\hline Imipenem & $1( \pm 0.47)$ & $0.066( \pm 0.01)$ & $0.58( \pm 0.084)$ & $0.19( \pm 0.043)$ & 1.72 & 0.34 \\
\hline
\end{tabular}

\footnotetext{
${ }^{a}$ Data for OXA-24 are from reference 21 .
}

${ }^{b} \mathrm{NT}$, not tested. 
ally (8). Because OXA-236 and OXA-237 were identified in single isolates while OXA-235 was identified in eight isolates, we propose that the subclass be termed OXA-235-like.

We found OXA-235-like variants encoded on plasmids isolated from multiple geographical regions, and they were able to transform into laboratory strains and reduce carbapenem susceptibility. Sequencing of $b l a_{\mathrm{OXA}-51-\text { like }}$ revealed that the majority of isolates possess OXA-66, which is associated with IC2, the most widespread A. baumannii lineage $(8,31)$. This strongly suggests that there is potential for further spread of OXA-235-like. Indeed, we found five OXA-235 isolates that showed $\geq 99 \%$ similarity by rep-PCR, suggesting clonal spread, as well as two variants of this enzyme, disseminated in 5 U.S. states and Mexico. This contrasts with OXA-143, which we found in isolates that did not cluster with the international clones, and may in part explain why this OXA has thus far been confined to Brazil (8). Additionally, the genes encoding OXA-235-like were undetected using established PCR methods, so it is possible that they have been overlooked in other studies. For example, isolate BMBF 255 (OXA-236) was part of a previous study into the global spread of carbapenem-resistant A. baumannii (8), but we were unable to identify the mechanism of resistance at that time, and it was only identified after designing primers for $b l a_{\text {OXA-235. }}$.

OXA-235 hydrolyzed penicillins and carbapenems but did not show activity against expanded-spectrum cephalosporins, as observed with other CHDLs $(25,27,30,32)$. Similar to the latter enzymes, OXA-235 shows high affinity for the carbapenems but with low rates of hydrolysis (low $k_{\text {cat }}$ ). Despite this weak hydrolysis, it is very likely that OXA-235 contributes significantly to imipenem and meropenem resistance, as demonstrated previously with OXA-23, OXA-24, OXA-58, and OXA-143, especially as $b l a_{\text {OXA-235-like }}$ is associated with ISAba1, which has been shown to promote overexpression of OXA (7).

The origin of acquired CHDLs associated with A. baumannii is not known, but recent evidence has emerged that OXA-23 is intrinsic to Acinetobacter radioresistens and has been mobilized, possibly by ISAbal (33). Other Acinetobacter species appear to have their own intrinsic OXA but, on the whole, are rarely associated with carbapenem resistance, despite their ability to hydrolyze carbapenems, possibly because expression is low $(26,34)$. As well as OXA-23, the intrinsic OXA-51-like of A. baumannii is associated with carbapenem resistance but only when overexpressed (ISAbal associated) (7). This reinforces the role of ISAbal and its importance in the genome plasticity of A. baumannii (35).

In summary, OXA-235, OXA-236, and OXA-237 are the first representatives of a novel subclass of CHDLs whose prevalence remains to be determined. It may, indeed, be quite prevalent, since resistance to carbapenems in A. baumannii has not always been associated with known carbapenemases and previously designed primers could not detect this novel gene.

\section{ACKNOWLEDGMENTS}

The contributions of P.G.H. and H.S. were supported by a grant from Bundesministerium für Bildung und Forschung (BMBF), Klinische Forschergruppe Infektiologie, Germany (BMBF grant 01KI0771). G.B. was funded by grants FIS PI081613, PS09/00687, and PI12/00552 from ISCIII.

\section{REFERENCES}

1. Peleg AY, Seifert H, Paterson DL. 2008. Acinetobacter baumannii: Emergence of a successful pathogen. Clin. Microbiol. Rev. 21:538-582.
2. Wareham DW, Bean DC, Khanna P, Hennessy EM, Krahe D, Ely A, Millar M. 2008. Bloodstream infection due to Acinetobacter spp: epidemiology, risk factors and impact of multi-drug resistance. Eur. J. Clin. Microbiol. Infect. Dis. 27:607-612.

3. Dijkshoorn L, Nemec A, Seifert H. 2007. An increasing threat in hospitals: multidrug-resistant Acinetobacter baumannii. Nat. Rev. Microbiol. 5:939-951.

4. Park YK, Jung SI, Park KH, Kim DH, Choi JY, Kim SH, Ko KS. 2012. Changes in antimicrobial susceptibility and major clones of Acinetobacter calcoaceticus-baumannii complex isolates from a single hospital in Korea over 7 years. J. Med. Microbiol. 61:71-79.

5. Gogou V, Pournaras S, Giannouli M, Voulgari E, Piperaki ET, Zarrilli R, Tsakris A. 2011. Evolution of multidrug-resistant Acinetobacter baumannii clonal lineages: a 10 year study in Greece (2000-09). J. Antimicrob. Chemother. 66:2767-2772.

6. Poirel L, Bonnin RA, Nordmann P. 2011. Genetic basis of antibiotic resistance in pathogenic Acinetobacter species. IUBMB Life 63:1061-1067.

7. Turton JF, Ward ME, Woodford N, Kaufmann ME, Pike R, Livermore DM, Pitt TL. 2006. The role of ISAbal in expression of OXA carbapenemase genes in Acinetobacter baumannii. FEMS Microbiol. Lett. 258:72-77.

8. Higgins PG, Dammhayn C, Hackel M, Seifert H. 2010. Global spread of carbapenem-resistant Acinetobacter baumannii. J. Antimicrob. Chemother. 65:233-238.

9. Poirel L, Naas T, Nordmann P. 2010. Diversity, epidemiology, and genetics of class D beta-lactamases. Antimicrob. Agents Chemother. 54: 24-38.

10. Woodford N, Ellington MJ, Coelho JM, Turton JF, Ward ME, Brown S, Amyes SGB, Livermore DM. 2006. Multiplex PCR for genes encoding prevalent OXA carbapenemases in Acinetobacter spp. Int. J. Antimicrob. Agents 27:351-353.

11. Higgins PG, Lehmann M, Seifert H. 2010. Inclusion of OXA-143 primers in a multiplex polymerase chain reaction (PCR) for genes encoding prevalent OXA carbapenemases in Acinetobacter spp. Int. J. Antimicrob. Agents 35:305.

12. Higgins PG, Wisplinghoff H, Krut O, Seifert H. 2007. A PCR-based method to differentiate between Acinetobacter baumannii and Acinetobacter genomic species 13TU. Clin. Microbiol. Infect. 13:1199-1201.

13. Healy M, Huong J, Bittner T, Lising M, Frye S, Raza S, Schrock R, Manry J, Renwick A, Nieto R, Woods C, Versalovic J, Lupski JR. 2005. Microbial DNA typing by automated repetitive-sequence-based PCR. J. Clin. Microbiol. 43:199-207.

14. Ellington MJ, Kistler J, Livermore DM, Woodford N. 2007. Multiplex PCR for rapid detection of genes encoding acquired metallo-betalactamases. J. Antimicrob. Chemother. 59:321-322.

15. Pfeifer Y, Wilharm G, Zander E, Wichelhaus TA, Göttig S, Hunfeld KP, Seifert H, Witte W, Higgins PG. 2011. Molecular characterization of bla NDM-1 $_{1}$ in an Acinetobacter baumannii strain isolated in Germany in 2007. J. Antimicrob. Chemother. 66:1998-2001.

16. Heritier C, Poirel L, Fournier PE, Claverie JM, Raoult D, Nordmann P. 2005. Characterization of the naturally occurring oxacillinase of Acinetobacter baumannii. Antimicrob. Agents Chemother. 49:4174-4179.

17. Choi KH, Kumar A, Schweizer HP. 2006. A 10-min method for preparation of highly electrocompetent Pseudomonas aeruginosa cells: application for DNA fragment transfer between chromosomes and plasmid transformation. J. Microbiol. Methods 64:391-397.

18. Kovach ME, Phillips RW, Elzer PH, Roop RM, Peterson KM. 1994. pBBR1MCS - a broad-host-range cloning vector. Biotechniques 16: $800-802$.

19. Bertini A, Poirel L, Mugnier PD, Villa L, Nordmann P, Carattoli A. 2010. Characterization and PCR-based replicon typing of resistance plasmids in Acinetobacter baumannii. Antimicrob. Agents Chemother. 54: 4168-4177.

20. Aranda J, Poza M, Pardo BG, Rumbo S, Rumbo C, Parreira JR, Rodriguez-Velo P, Bou G. 2010. A rapid and simple method for constructing stable mutants of Acinetobacter baumannii. BMC Microbiol. 10: 279. doi:10.1186/1471-2180-10-279.

21. Santillana E, Beceiro A, Bou G, Romero A. 2007. Crystal structure of the carbapenemase OXA-24 reveals insights into the mechanism of carbapenem hydrolysis. Proc. Natl. Acad. Sci. U. S. A. 104:5354-5359.

22. Perez-Llarena FJ, Cartelle M, Mallo S, Beceiro A, Perez A, Villanueva R, Romero A, Bonnet R, Bou G. 2008. Structure-function studies of argi- 
nine at position 276 in CTX-M beta-lactamases. J. Antimicrob. Chemother. 61:792-797.

23. Zander E, Nemec A, Seifert H, Higgins PG. 2012. Association between $\beta$-lactamase-encoding $b l a_{\text {OXA-51 }}$ variants and DiversiLab rep-PCR-based typing of Acinetobacter baumannii isolates. J. Clin. Microbiol. 50:19001904.

24. Couture F, Lachapelle J, Levesque RC. 1992. Phylogeny of LCR-1 and OXA-5 with class-A and class-D beta-lactamases. Mol. Microbiol. 6:16931705.

25. Poirel L, Marque S, Heritier C, Segonds C, Chabanon G, Nordmann P. 2005. OXA-58, a novel class D beta-lactamase involved in resistance to carbapenems in Acinetobacter baumannii. Antimicrob. Agents Chemother. 49:202-208.

26. Figueiredo S, Poirel L, Seifert H, Mugnier P, Benhamou D, Nordmann P. 2010. OXA-134, a naturally occurring carbapenem-hydrolyzing class D beta-lactamase from Acinetobacter lwoffii. Antimicrob. Agents Chemother. 54:5372-5375.

27. Higgins PG, Poirel L, Lehmann M, Nordmann P, Seifert H. 2009. OXA-143, a novel carbapenem-hydrolyzing class D $\beta$-lactamase in Acinetobacter baumannii. Antimicrob. Agents Chemother. 53:5035-5038.

28. Antonio CS, Neves PR, Medeiros M, Mamizuka EM, Elmor de Araujo MR, Lincopan N. 2011. High prevalence of carbapenem-resistant Acinetobacter baumannii carrying the bla ${ }_{\mathrm{OXA}-143}$ gene in Brazilian hospitals. Antimicrob. Agents Chemother. 55:1322-1323.

29. Scaife W, Young HK, Paton RH, Amyes SGB. 1995. Transferable imi- penem-resistance in Acinetobacter species from a clinical source. J. Antimicrob. Chemother. 36:585-586.

30. Bou G, Oliver A, Martinez-Beltran J. 2000. OXA-24, a novel class D beta-lactamase with carbapenemase activity in an Acinetobacter baumannii clinical strain. Antimicrob. Agents Chemother. 44:1556-1561.

31. Turton JF, Gabriel SN, Valderrey C, Kaufmann ME, Pitt TL. 2007. Use of sequence-based typing and multiplex PCR to identify clonal lineages of outbreak strains of Acinetobacter baumannii. Clin. Microbiol. Infect. 13: $807-815$.

32. Afzal-Shah M, Woodford N, Livermore DM. 2001. Characterization of OXA-25, OXA-26, and OXA-27, molecular class D beta-lactamases associated with carbapenem resistance in clinical isolates of Acinetobacter baumannii. Antimicrob. Agents Chemother. 45:583-588.

33. Poirel L, Figueiredo S, Cattoir V, Carattoli A, Nordmann P. 2008. Acinetobacter radioresistens as a silent source of carbapenem resistance for Acinetobacter spp. Antimicrob. Agents Chemother. 52:1252-1256.

34. Figueiredo S, Bonnin RA, Poirel L, Duranteau J, Nordmann P. 2012. Identification of the naturally occurring genes encoding carbapenemhydrolysing oxacillinases from Acinetobacter haemolyticus, Acinetobacter johnsonii, and Acinetobacter calcoaceticus. Clin. Microbiol. Infect. 18:907913.

35. Mugnier PD, Poirel L, Nordmann P. 2009. Functional analysis of insertion sequence ISAba1, responsible for genomic plasticity of Acinetobacter baumannii. J. Bacteriol. 191:2414-2418. 